\title{
Self-Reconstruction Property of a Special Speckle Pattern after Obstructing by an Opaque Obstruction
}

\author{
Yanyu Chen, Huang Tan, and Gaofeng Wu \\ School of Physics, Northwest University, Xian 710069, China \\ Correspondence should be addressed to Gaofeng Wu; gfwu@nwu.edu.cn
}

Received 29 June 2017; Accepted 21 August 2017; Published 26 September 2017

Academic Editor: Shuqing Chen

Copyright (c) 2017 Yanyu Chen et al. This is an open access article distributed under the Creative Commons Attribution License, which permits unrestricted use, distribution, and reproduction in any medium, provided the original work is properly cited.

\begin{abstract}
Numerical simulation of random optical field with special autocorrelation is performed. It can be seen that the speckle pattern with special autocorrelation is different from the general speckle pattern whose autocorrelation is a Gaussian function. Furthermore, we study the propagation property of the special speckle in free space after blocking by an opaque obstruction and find the self-healing effect of speckle. In particular, we investigate the influence of size and shape of obstruction and the coherence of speckle on the speckle's reconstruction process. We find that it is affected by the size of obstruction and the coherence of the speckle but is almost not affected by the shape of obstruction. Our results can be applied to the imaging, optical communication, and so on.
\end{abstract}

\section{Introduction}

Random scatting strongly distorts the optical fields, creating the well-known speckle pattern. The speckle pattern is formed by superposing all the random optical fields. In past times, speckle pattern is harmful to the optical imaging, optical communication, and so on. People usually avoid producing speckle. However, in recent years, random scattering has emerged as a rich research field. This is because it has useful application. The scattering field can form a subwavelength focal spot by modulating the wavefront $[1,2]$. Disordered scattering has been applied to improve imaging resolution [3-5]. Noninvasive imaging of a fluorescent object has been done by measuring the autocorrelation of the speckle [6]. The biological imaging using speckle scanning microscopy was studied [7].

There are some special light beams, called nondiffraction beam, can keep its beam profile during propagation, like Bessel beams [8], Airy beams [9], and so on. They can be self-healing when those beams are partially blocked by an opaque obstruction. Hence, they have been widely applied to microscopic particle manipulation [10], human tissue microscopy [11], quantum entanglement propagation in the presence of obstructions [12], and optical communications through inhomogeneous media [13, 14]. In 2014, Alves et al. found that the speckle showed a robust self-healing property [15], but they only paid attention to the speckle generated by the Gaussian beam and the Bessel beam. In the present paper, we will simulate the special speckle generated by the hyperbolic cosine-Gaussian beam and investigate its propagation after blocking by an opaque obstruction.

\section{Simulation of the Speckle Generation}

Speckle pattern is the random intensity distribution. The numerical simulation of the speckle can follow Goodman's book [16]. We use a matrix with $512 \times 512$ pixels and every pixel size is $0.097656 \mathrm{~mm}$. First, a matrix of random phasors was generated and then multiplied by the incident beam. The incident beam can be Gaussian, Bessel, or other kinds of beams. Here, we use the hyperbolic cosine-Gaussian function as the incident beam

$$
\begin{aligned}
I\left(f_{x}, f_{y}\right)= & 2 \pi \delta^{2} \cosh \left(n \sqrt{2 \pi} \delta f_{x}\right) \cosh \left(n \sqrt{2 \pi} \delta f_{y}\right) \\
& \cdot \exp \left(-\frac{\delta^{2}\left(f_{x}^{2}+f_{y}^{2}\right)+2 n^{2} \pi}{2}\right),
\end{aligned}
$$

where $n$ and $\delta$ are positive real constants. $\cosh (x)$ is the hyperbolic cosine function. After that, we can get the speckle 


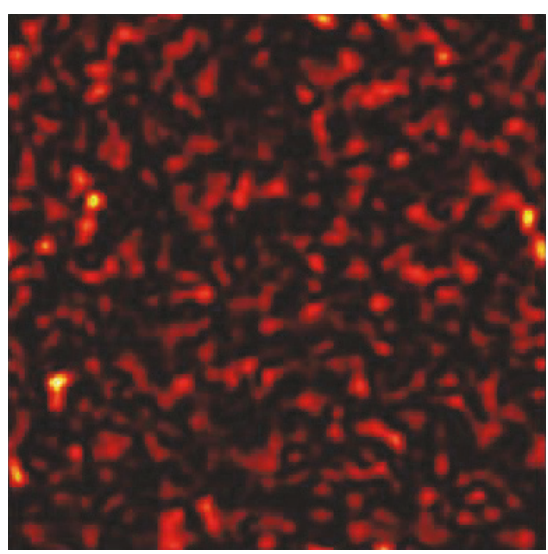

(a)

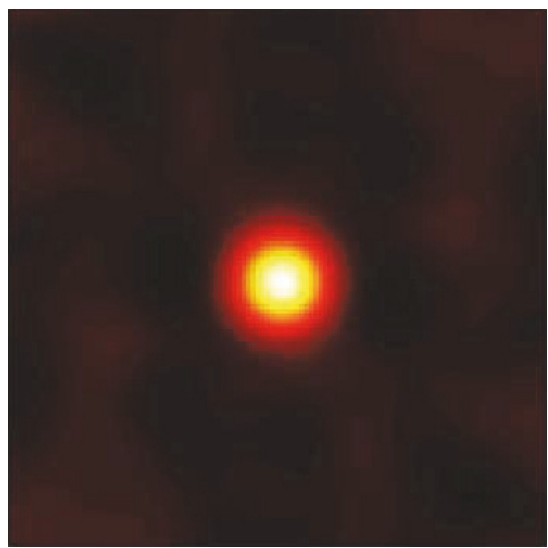

(d)

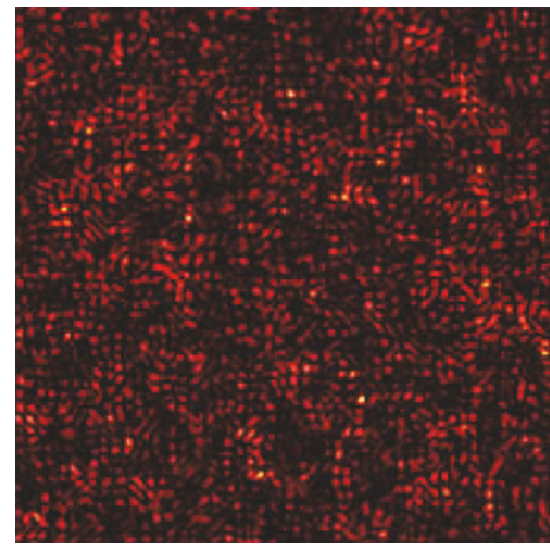

(b)

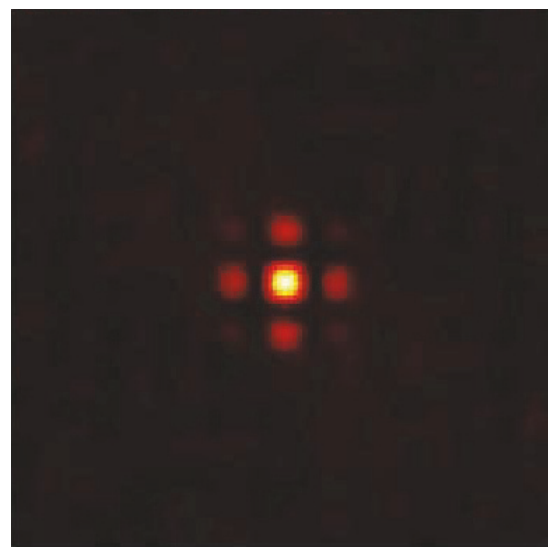

(e)

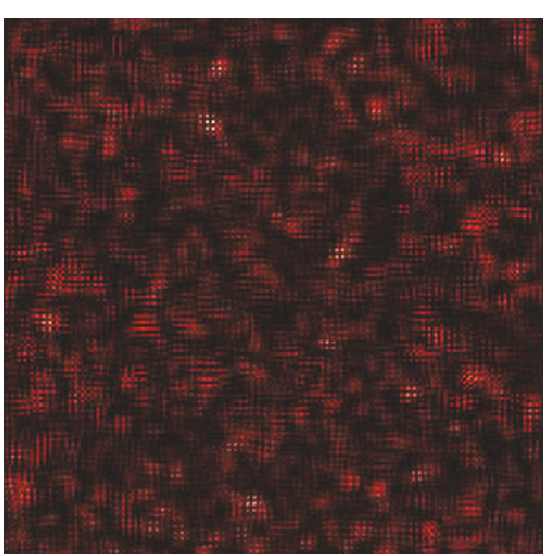

(c)

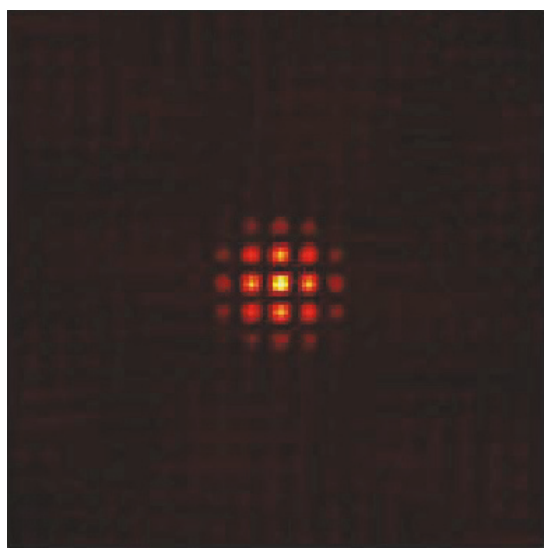

(f)

FIGURE 1: The simulation of speckle pattern in the source plane for different $n$ with $\delta=6 \mathrm{~mm}$, (a) $n=0$, (b) $n=1$, and (c) $n=2$ and the autocorrelation corresponding to the speckle pattern, (d) $n=0$, (e) $n=1$, and (f) $n=2$.

by performing the fast Fourier transform of the resulting matrix. Figure 1 shows the speckle pattern for selected value of $n$. One can find that the parameter $n$ affects the speckle distribution. We obtain the ordinary speckle, that is, Gaussian speckle, when $n=0$, and the special speckle pattern when $n \neq 0$. The difference among those patterns can be found from the autocorrelation which is defined as

$$
\begin{aligned}
\operatorname{AC} & (\Delta x, \Delta y) \\
= & \iint I_{\text {out }}(x, y) I_{\text {out }}(x-\Delta x, y-\Delta y) d x d y,
\end{aligned}
$$

where $I_{\text {out }}(x, y)=E^{*}(x, y) E(x, y)$ is the speckle pattern, that is, the speckle intensity, and $E(x, y)$ is the electric field. For the Gaussian speckle, its autocorrelation is a Gaussian distribution [see Figure 1(a)], but the autocorrelation of those speckle with $n \neq 0$ is different. There is a spot in the center surrounded by other spots. The amount of the surrounded spots is large when the value of $n$ is large [see Figure 1(c)]. Figure 2 shows the speckle pattern and autocorrelation for different $\delta$. It is found that the average size of the speckle (speckle- coherence length) increases as the value of $\delta$ increases. That is also reflected by the pattern of autocorrelation. It is big with a large value of $\delta$. If we change the value of $\delta$ with different $n$, one can see the average size of the speckle pattern change. It is also found that the size of the autocorrelation varies as the value of $\delta$ changes. In fact, the inverse of $\delta$ is related to the incident beam size [17]. Therefore, we can control the average size of the speckle by varying the beam size of the incident beam.

\section{The Propagation of Speckle}

The propagation of the speckle can be expressed by the Huygens-Fresnel integral as

$$
\begin{gathered}
E_{2}\left(x_{2}, y_{2}, z\right)=\left(-\frac{i}{\lambda z}\right) \exp (i k z) \iint E_{1}\left(x_{1}, y_{1}, 0\right) \\
\times \exp \left\{\frac{i k}{2 z}\left[\left(x_{2}-x_{1}\right)^{2}+\left(y_{2}-y_{1}\right)^{2}\right]\right\} d x_{1} d y_{1},
\end{gathered}
$$

where $E_{1}\left(x_{1}, y_{1}, 0\right)$ and $E_{2}\left(x_{2}, y_{2}, z\right)$ are the random electric field in the source plane and received plane, respectively. $k=$ $2 \pi / \lambda$ denotes the wavenumber with wavelength $\lambda . x_{1}, y_{1}$ and 


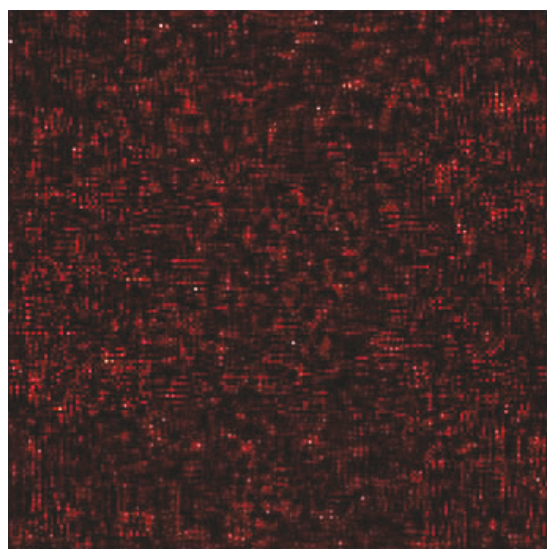

(a)

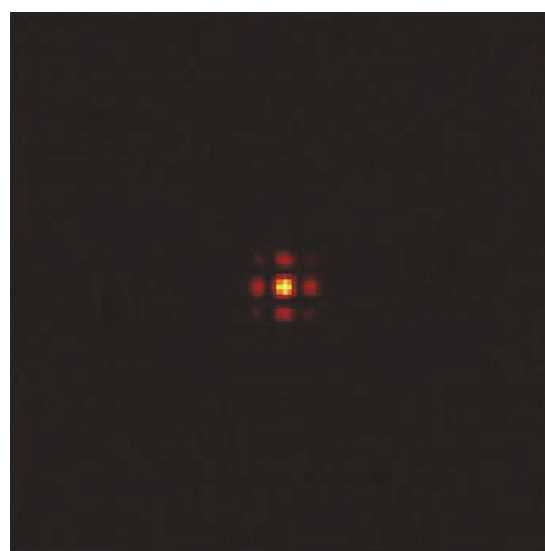

(d)

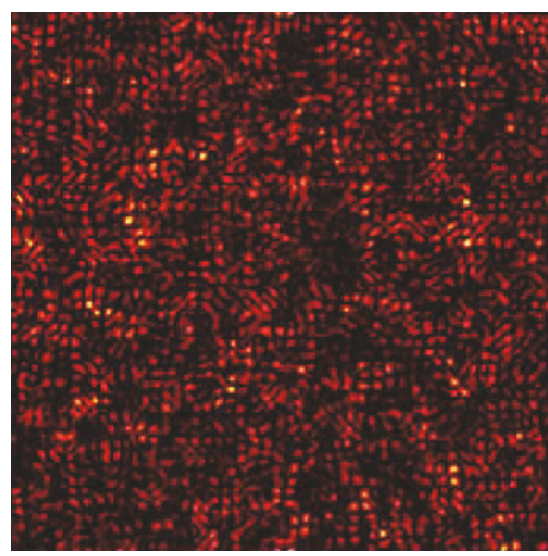

(b)

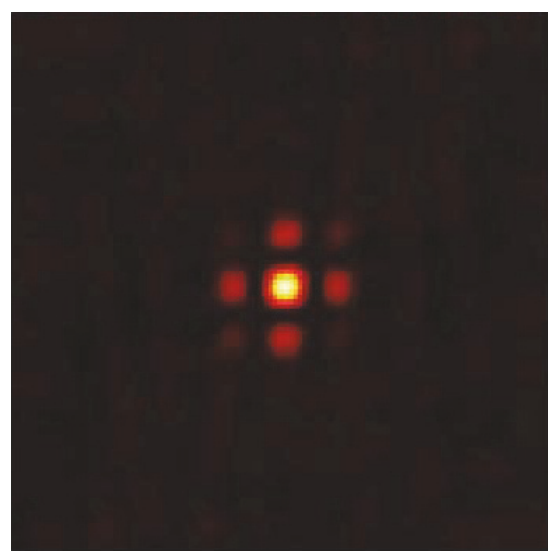

(e)

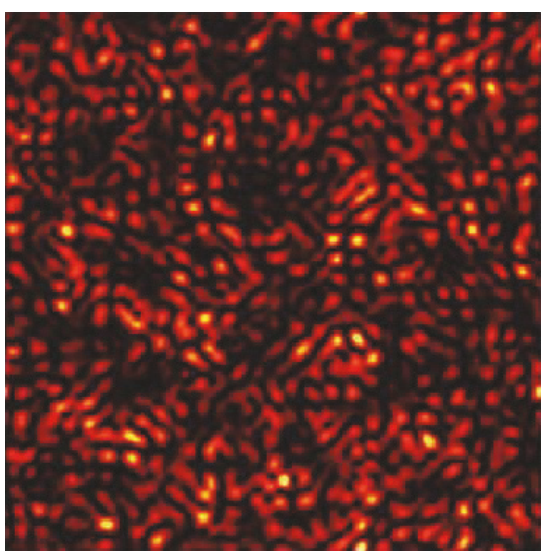

(c)

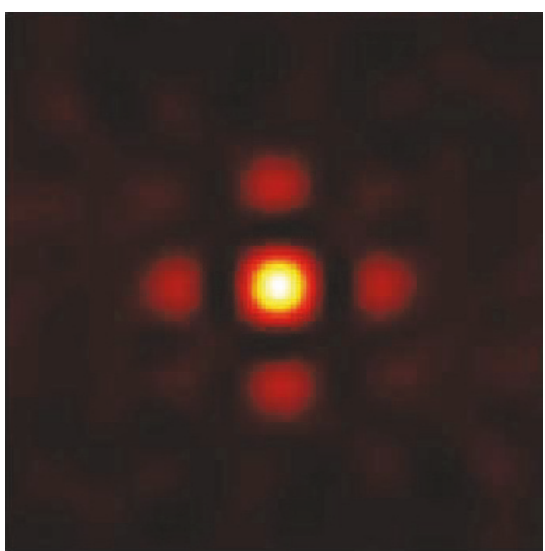

(f)

FIGURE 2: The simulation of speckle pattern in the source plane and corresponding to the autocorrelation for different $\delta$ with $n=1$, (a), (d) $\delta=3 \mathrm{~mm}$, (b), (e) $\delta=6 \mathrm{~mm}$, and (c), (f) $\delta=12 \mathrm{~mm}$.

$x_{2}, y_{2}$ are the transverse coordinates in the source plane and received plane. Equation (3) can be written in an altered form as

$$
\begin{gathered}
E_{2}\left(x_{2}, y_{2}, z\right)=\left(-\frac{i}{\lambda z}\right) \exp (i k z) \exp \left[\frac{i k}{2 z}\left(x_{2}^{2}+y_{2}^{2}\right)\right] \\
\cdot \iint E_{1}\left(x_{1}, y_{1}, 0\right) \exp \left[\frac{i k}{2 z}\left(x_{1}^{2}+y_{1}^{2}\right)\right] \\
\cdot \exp \left[-\frac{i k}{z}\left(x_{2} x_{1}+y_{2} y_{1}\right)\right] d x_{1} d y_{1} .
\end{gathered}
$$

One can find from (4) that the integral is a Fourier transform. Namely,

$$
\begin{aligned}
E_{2}\left(x_{2}, y_{2}, z\right) & \\
= & \left(-\frac{i}{\lambda z}\right) \exp (i k z) \exp \left[\frac{i k}{2 z}\left(x_{2}^{2}+y_{2}^{2}\right)\right] \\
& \times \widetilde{F}\left\{E_{1}\left(x_{1}, y_{1}, 0\right) \exp \left[\frac{i k}{2 z}\left(x_{1}^{2}+y_{1}^{2}\right)\right]\right\} .
\end{aligned}
$$

The Fourier transform can be easily performed by the fast Fourier transform algorithm. If the speckle in the source plane is blocked by an opaque obstruction, the propagation of the missing speckle can be expressed as

$$
\begin{aligned}
& E_{2}\left(x_{2}, y_{2}, z\right)=\left(-\frac{i}{\lambda z}\right) \exp (i k z) \exp \left[\frac{i k}{2 z}\left(x_{2}^{2}+y_{2}^{2}\right)\right] \\
& \times \widetilde{F}\left\{E_{1}\left(x_{1}, y_{1}, 0\right) T\left(x_{1}, y_{1}, 0\right)\right. \\
& \left.\cdot \exp \left[\frac{i k}{2 z}\left(x_{1}^{2}+y_{1}^{2}\right)\right]\right\},
\end{aligned}
$$

where $T\left(x_{1}, y_{1}, 0\right)$ denotes the transmission function of the obstruction. When $T\left(x_{1}, y_{1}, 0\right)=1$ for every point in the source plane, the random filed $E_{1}\left(x_{1}, y_{1}, 0\right)$ does not been modulated by the obstruction. That means there is no obstruction. When $T\left(x_{1}, y_{1}, 0\right)=0$ which means the random field is completely blocked by the obstruction, the speckle can not propagate. We will investigate the situation that the speckle is partially blocked by the obstruction. The 


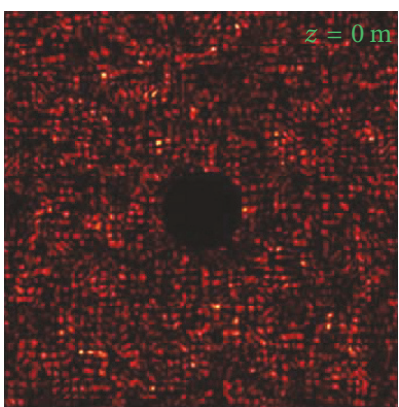

(a)

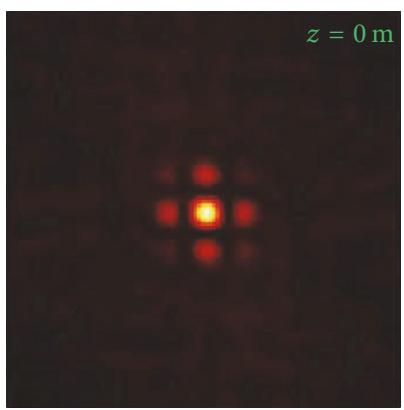

(e)

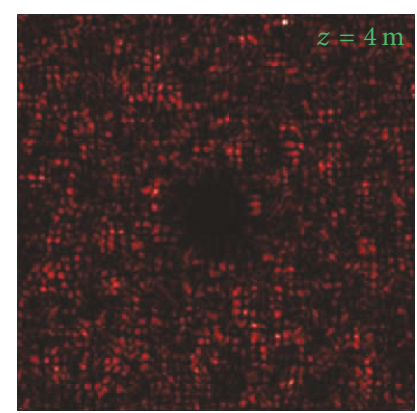

(b)

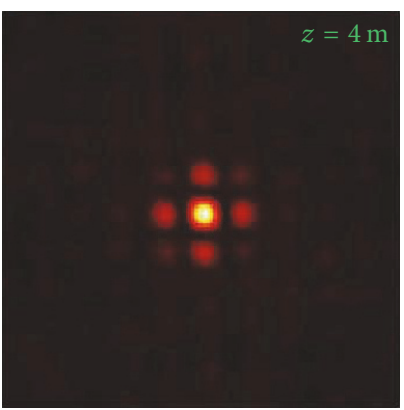

(f)

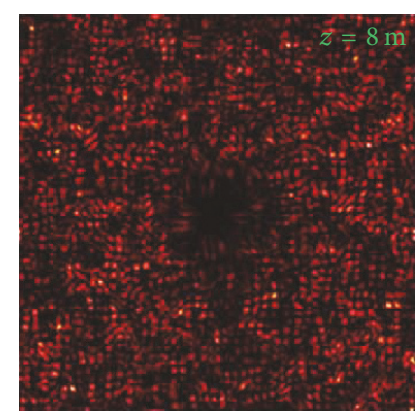

(c)

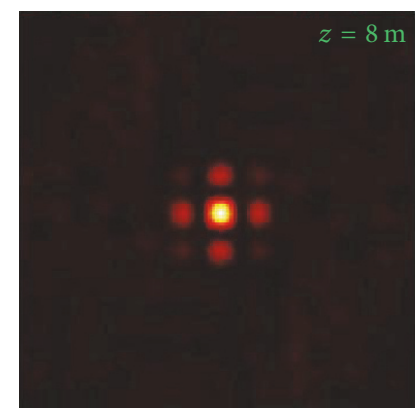

(g)

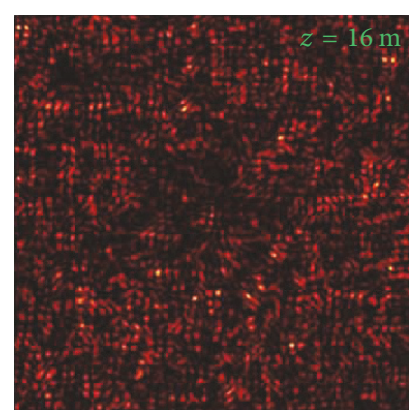

(d)

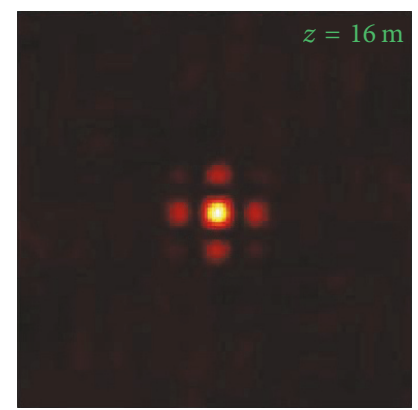

(h)

Figure 3: (a)-(d) The simulation of speckle pattern at different propagating distances with $R=5 \mathrm{~mm}, \delta=6 \mathrm{~mm}$, and $n=1$. (e)-(h) corresponding to the distribution of the autocorrelation.

wavelength $\lambda$ is $632.8 \mathrm{~nm}$. The obstruction is an opaque disk with radius $R=5 \mathrm{~mm}$.

From Figure 3, one can find that the speckle pattern in the source plane is blocked by an opaque disk in the center. Therefore, one can find a black hole in the center. However, the hole gradually disappears as the speckle propagates in free space. When the speckle propagates to the plane $z=16 \mathrm{~m}$, one almost cannot find the hole in the center. That means the speckle can reconstruct itself as it propagates in free space. On the other hand, it is found that the autocorrelation at every plane is almost the same. In other words, the autocorrelation almost is not affected by the obstruction.

The influence of the size of obstruction on the reconstruction of speckle is showed in Figure 4. The first row shows the speckle in the source plane with different size of obstruction. The second row shows the speckle pattern in the plane of $z=$ $16 \mathrm{~m}$. One can find that the speckle with a small obstruction can primly reconstruct itself, whereas the speckle with a large obstruction does not at the same propagating distance. It needs long distance to diffract and to finish its reconstruction. The third row shows the autocorrelation in the plane of $z=$ $16 \mathrm{~m}$. It is found that the autocorrelation distribution is hardly affected by the size of the obstruction. Figure 5 shows the influence of the shape of obstruction on the reconstruction of speckle pattern and on autocorrelation. The obstruction has the same area but with different shape. It is found that the shape does not affect the reconstruction of speckle pattern. Although the pattern is partially blocked by different shape of obstruction, it can be self-healing as the speckle propagates in free space. As showed in the second row of Figure 5, all the speckle patterns finished their reconstruction process and one cannot find the obvious hole in the center. One can also find that the autocorrelation of the speckle pattern in the plane of $z=16 \mathrm{~m}$ is the same. They keep the same shape as it in the source plane. Figure 6 shows the speckle pattern at different propagating distance for different coherence length. One can find that there is a circle hole in the center of the speckle pattern with the coherence length $\delta=3 \mathrm{~mm}$ in the source plane. This is because the opaque obstruction partially blocks the speckle. When the speckle propagates to the plane of $z=4 \mathrm{~m}$, one can still find the hole. However, there is no obvious hole when the speckle propagates to the plane of $z=8 \mathrm{~m}$. That means the speckle almost finished its reconstruction process when it propagates to the plane of $z=8 \mathrm{~m}$. However, in the second row, one can find that there is a hole in the center when the speckle propagates to the same distance $z=8 \mathrm{~m}$ and it disappears at the plane of $z=16 \mathrm{~m}$. In the third row, one can find that the hole disappears at the plane of $z=28 \mathrm{~m}$. Those imply that the speckles with a high coherence need a long propagating distance to reconstruct themselves.

\section{Conclusions}

We have simulated the laser speckle with special autocorrelation and investigated its propagation in free space after partial blocking by an opaque obstruction. It was found that the missing speckle pattern can reconstruct itself and 


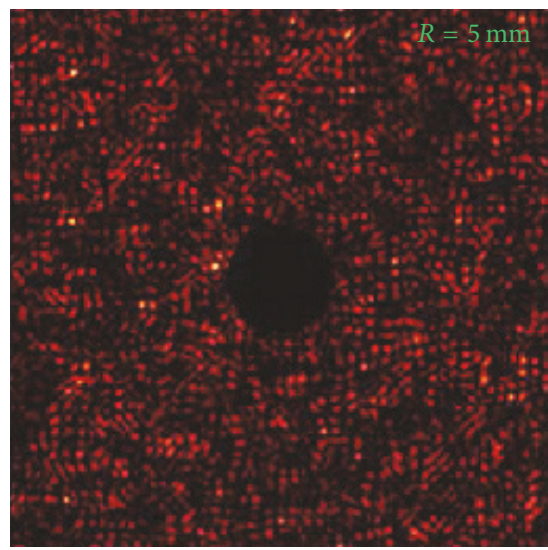

(a1)

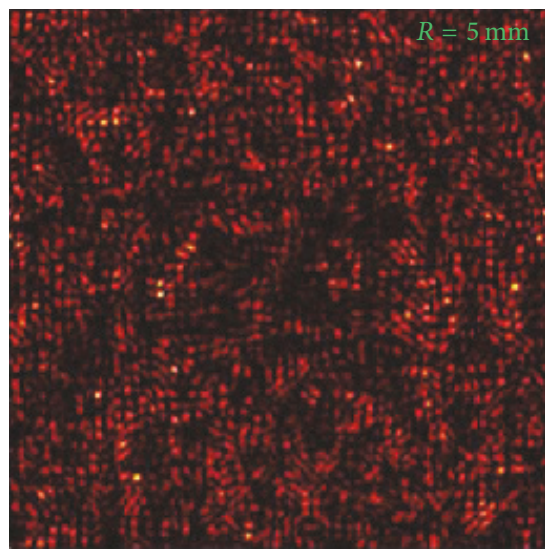

(a2)

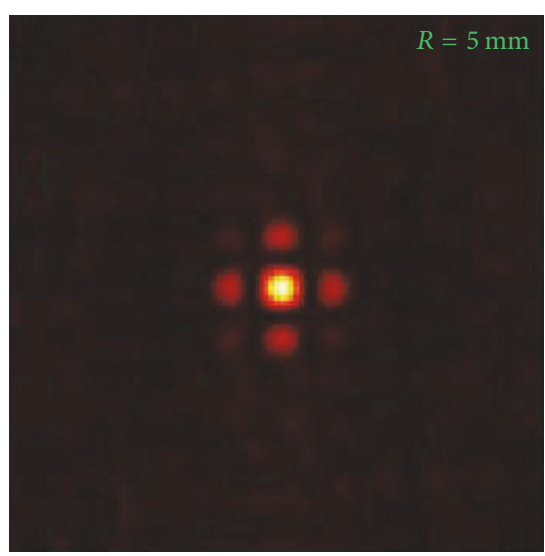

(a3)

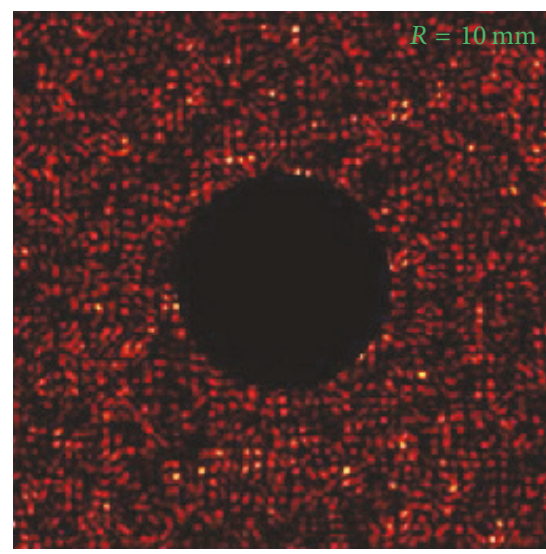

(b1)

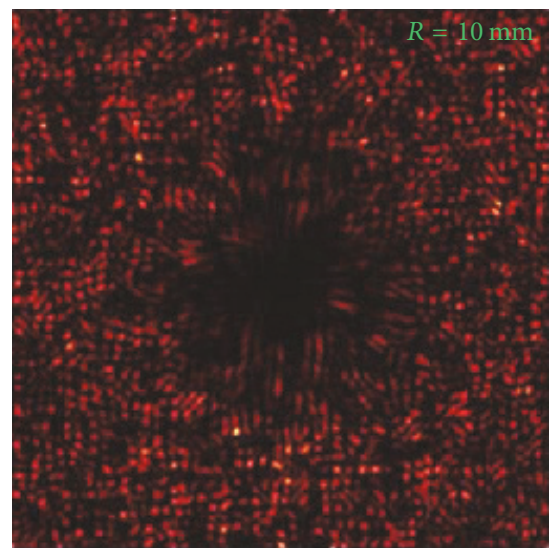

(b2)

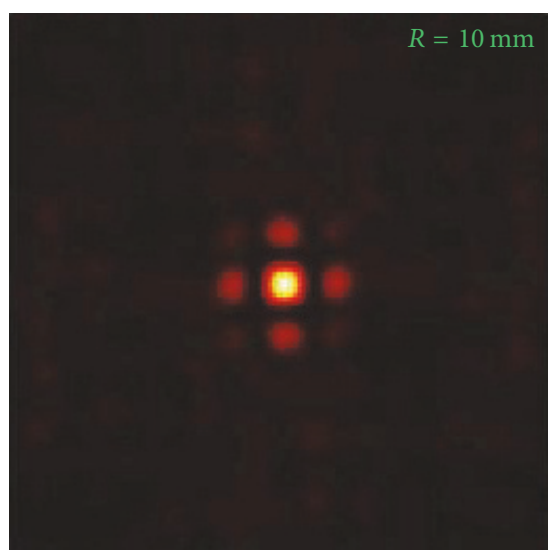

(b3)

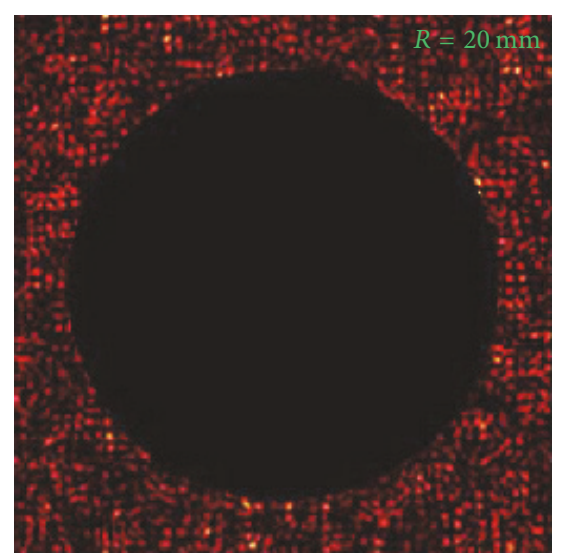

(c1)

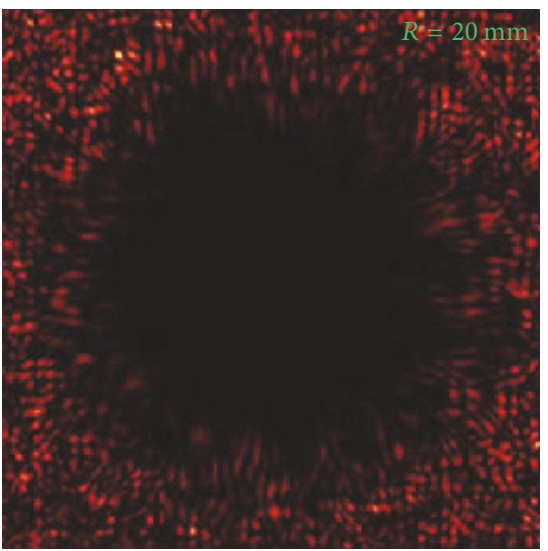

(c2)

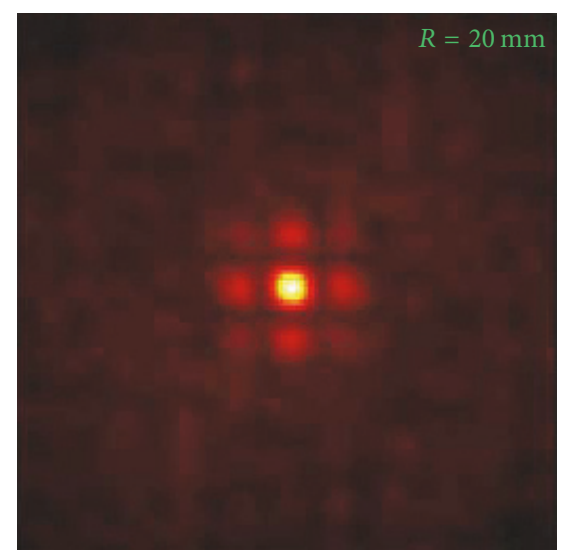

(c3)

FIGURE 4: The simulation of speckle pattern and corresponding to the autocorrelation for different size of obstruction with $\delta=6 \mathrm{~mm} ; n=1$. The first row denotes the speckle pattern in the source plane, the second row denotes the speckle pattern in the plane of $z=16 \mathrm{~m}$, and the third row denotes the autocorrelation of the speckle pattern in the second row.

its autocorrelation kept the same shape as it propagated in free space. The reconstruction of the speckle is not affected by the shape of the obstruction but relates to the size of the obstruction. The speckle needs a long distance to reconstruct itself when the size of the obstruction is large. We also found the influence of coherence of speckle on its reconstruction. The speckle needs a long propagating distance to reconstruct when the coherence of the speckle is high. It is also found that the size and shape of the obstruction have a little influence on the autocorrelation. This implies that we can use the autocorrelation to resist disturbance and load information. 


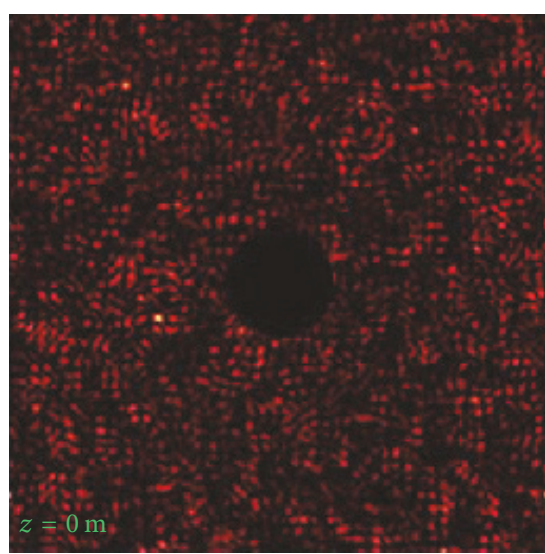

(a1)

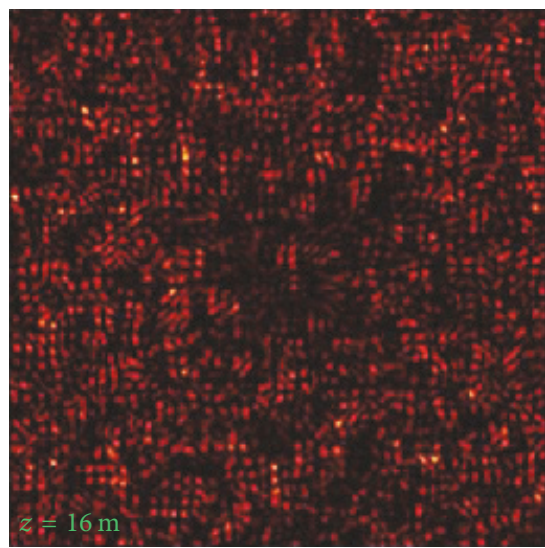

(a2)

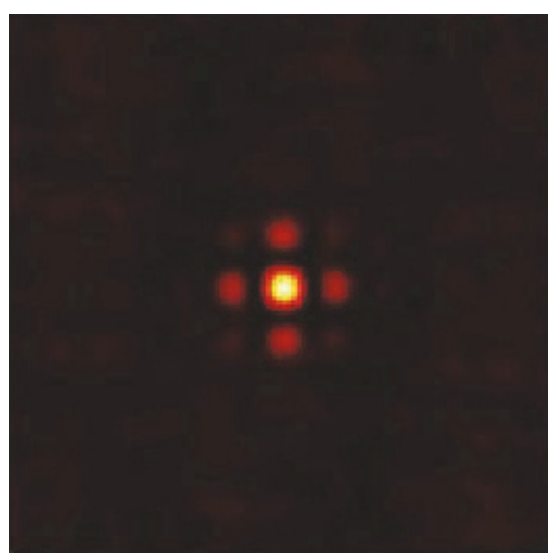

(a3)

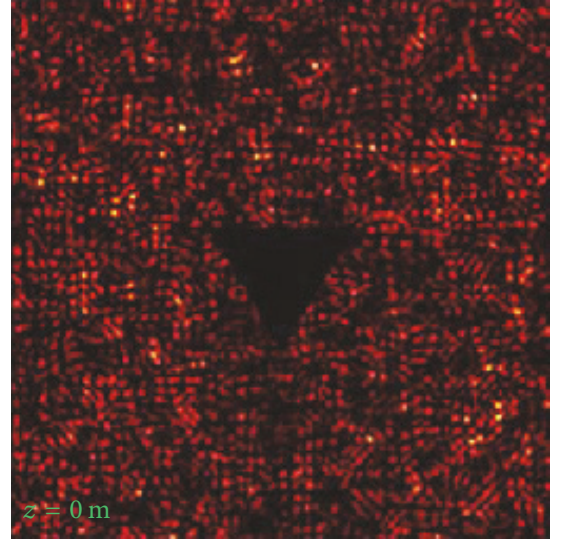

(b1)

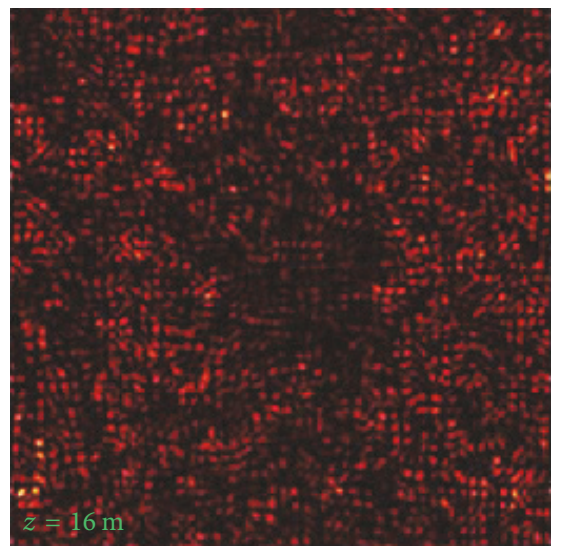

(b2)

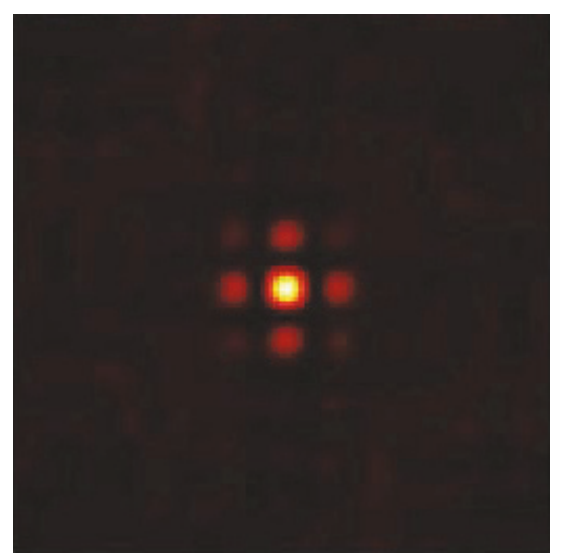

(b3)

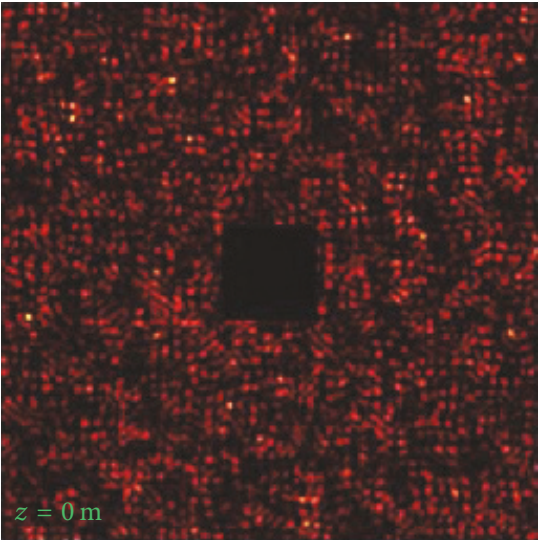

(c1)

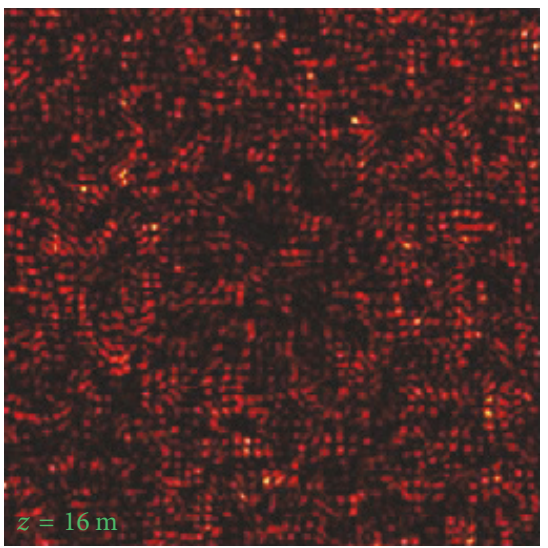

(c2)

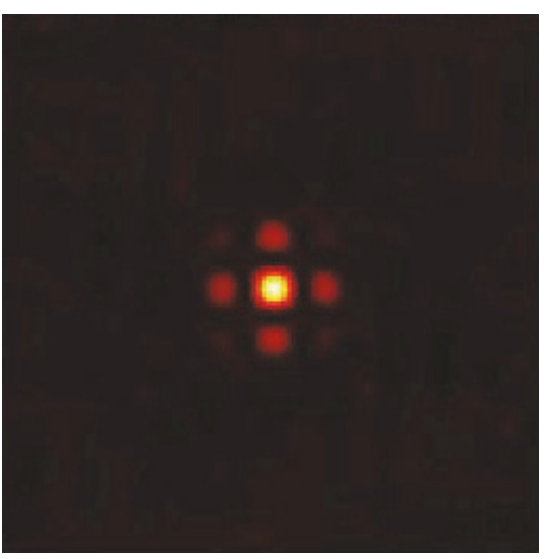

(c3)

FIGURE 5: The simulation of speckle pattern and corresponding to the autocorrelation for different shape of obstruction with $\delta=6 \mathrm{~mm} ; n=1$. The shape of the obstruction is circle (al), equilateral triangle (b1) and square (c1). The first row denotes the speckle pattern in the source plane, the second row denotes the speckle pattern in the plane of $z=16 \mathrm{~m}$, and the third row denotes the autocorrelation of the speckle pattern in the second row.

\section{Conflicts of Interest}

The authors declare that there are no conflicts of interest regarding the publication of this paper.

\section{Acknowledgments}

This work is supported by Natural Science Basic Research Plan in Shaanxi Province of China (Program no. 2016JQ1021); 


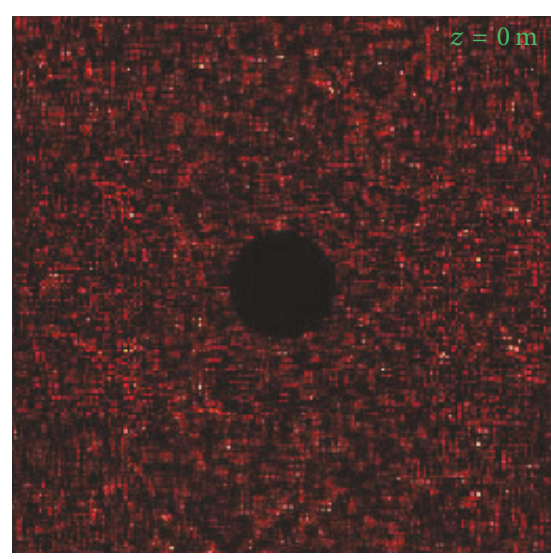

(a1)

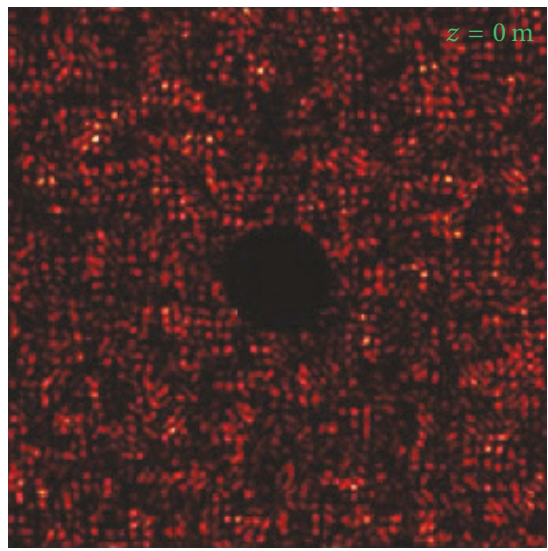

(b1)

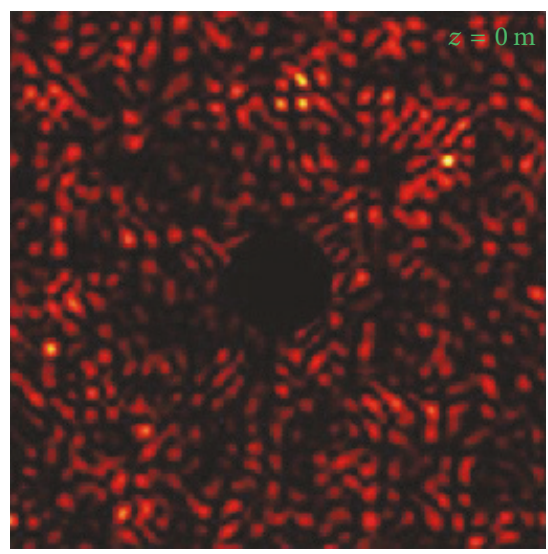

(c1)

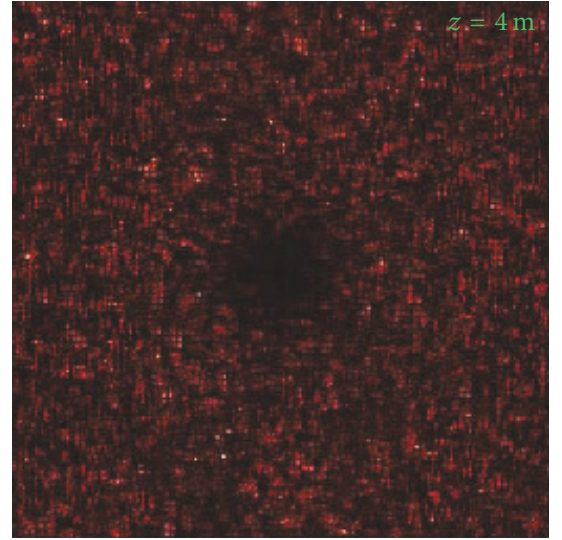

(a2)

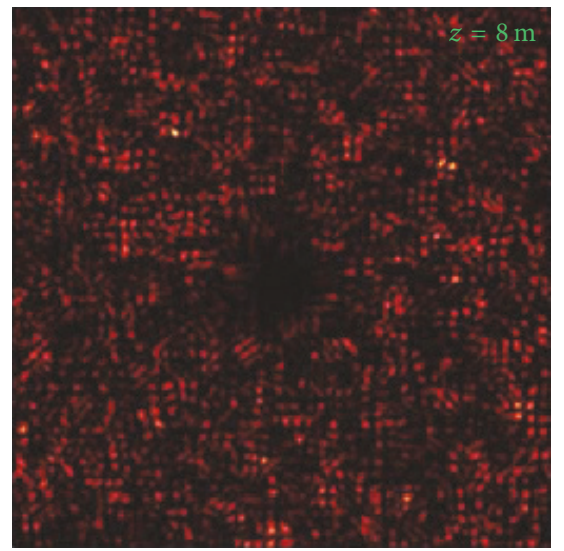

(b2)

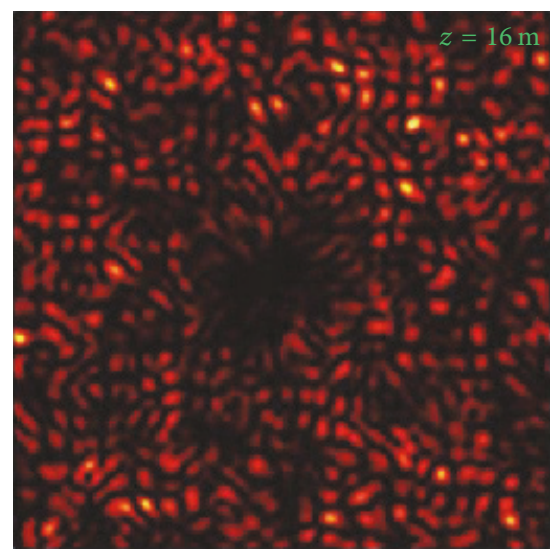

(c2)

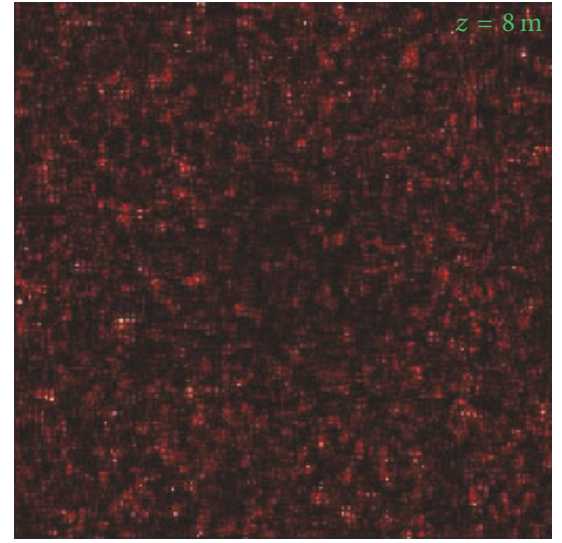

(a3)

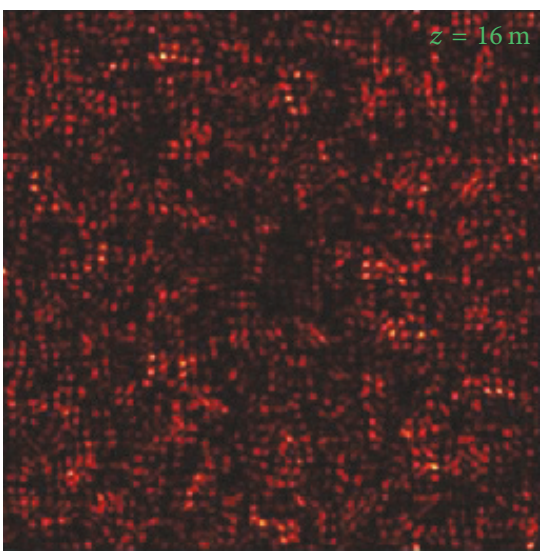

(b3)

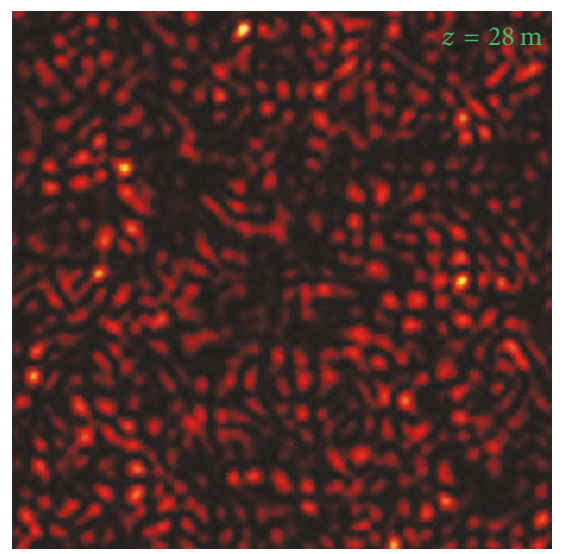

(c3)

FIGURE 6: The simulation of speckle pattern at different propagating distance for different coherence length with $R=5 \mathrm{~mm}, n=1$. The first row $\delta=3 \mathrm{~mm}$, the second row $\delta=6 \mathrm{~mm}$, and the third row $\delta=12 \mathrm{~mm}$.

the National Natural Sciences Foundation of China (NSFC) (11604264); Science Foundation of Northwest University (15NW28).

\section{References}

[1] I. M. Vellekoop, A. Lagendijk, and A. P. Mosk, "Exploiting disorder for perfect focusing," Nature Photonics, vol. 4, no. 5, pp. 320-322, 2010.
[2] O. Katz, E. Small, Y. Bromberg, and Y. Silberberg, "Focusing and compression of ultrashort pulses through scattering media," Nature Photonics, vol. 5, no. 6, pp. 372-377, 2011.

[3] I. M. Vellekoop and C. M. Aegerter, "Scattered light fluorescence microscopy: Imaging through turbid layers," Optics Letters, vol. 35 , no. 8, pp. 1245-1247, 2010.

[4] E. G. Van Putten, D. Akbulut, J. Bertolotti, W. L. Vos, A. Lagendijk, and A. P. Mosk, "Scattering lens resolves sub-100 nm 
structures with visible light," Physical Review Letters, vol. 106, no. 19, Article ID 193905, 2011.

[5] Y. Choi, T. D. Yang, C. Fang-Yen et al., "Overcoming the diffraction limit using multiple light scattering in a highly disordered medium," Physical Review Letters, vol. 107, no. 2, Article ID 023902, 2011.

[6] J. Bertolotti, E. G. Van Putten, C. Blum, A. Lagendijk, W. L. Vos, and A. P. Mosk, "Non-invasive imaging through opaque scattering layers," Nature, vol. 491, no. 7423, pp. 232-234, 2012.

[7] X. Yang, Y. Pu, and D. Psaltis, "Imaging blood cells through scattering biological tissue using speckle scanning microscopy," Optics Express, vol. 22, no. 3, pp. 3405-3413, 2014.

[8] J. Durnin, J. Miceli Jr., and J. H. Eberly, "Diffraction-free beams," Physical Review Letters, vol. 58, no. 15, pp. 1499-1501, 1987.

[9] G. A. Siviloglou, J. Broky, A. Dogariu, and D. N. Christodoulides, "Observation of accelerating airy beams," Physical Review Letters, vol. 99, no. 21, Article ID 213901, 2007.

[10] V. Garcés-Chávez, D. McGloin, H. Melville, W. Sibbett, and K. Dholakia, "Simultaneous micromanipulation in multiple planes using a self-reconstructing light beam," Nature, vol. 419, no. 6903, pp. 145-147, 2002.

[11] F. O. Fahrbach, P. Simon, and A. Rohrbach, "Microscopy with self-reconstructing beams," Nature Photonics, vol. 4, no. 11, pp. 780-785, 2010.

[12] M. McLaren, T. Mhlanga, M. J. Padgett, F. S. Roux, and A. Forbes, "Self-healing of quantum entanglement after an obstruction," Nature Communications, vol. 5, article no. 3248, 2014.

[13] J. Broky, G. A. Siviloglou, A. Dogariu, and D. N. Christodoulides, "Self-healing properties of optical Airy beams," Optics Express, vol. 16, no. 17, pp. 12880-12891, 2008.

[14] Y. Yuan, T. Lei, Z. Li et al., "Beam wander relieved orbital angular momentum communication in turbulent atmosphere using Bessel beams," Scientific Reports, vol. 7, Article ID 42276, 2017.

[15] C. R. Alves, A. J. Jesus-Silva, and E. J. S. Fonseca, "Selfreconfiguration of a speckle pattern," Optics Letters, vol. 39, no. 21, pp. 6320-6323, 2014.

[16] J. W. Goodman, Speckle Phenomena in Optics: Theory and Applications, Roberts, 2007.

[17] C. Liang, F. Wang, X. Liu, Y. Cai, and O. Korotkova, "Experimental generation of cosine-Gaussian-correlated Schell-model beams with rectangular symmetry," Optics Letters, vol. 39, no. 4, pp. 769-772, 2014. 

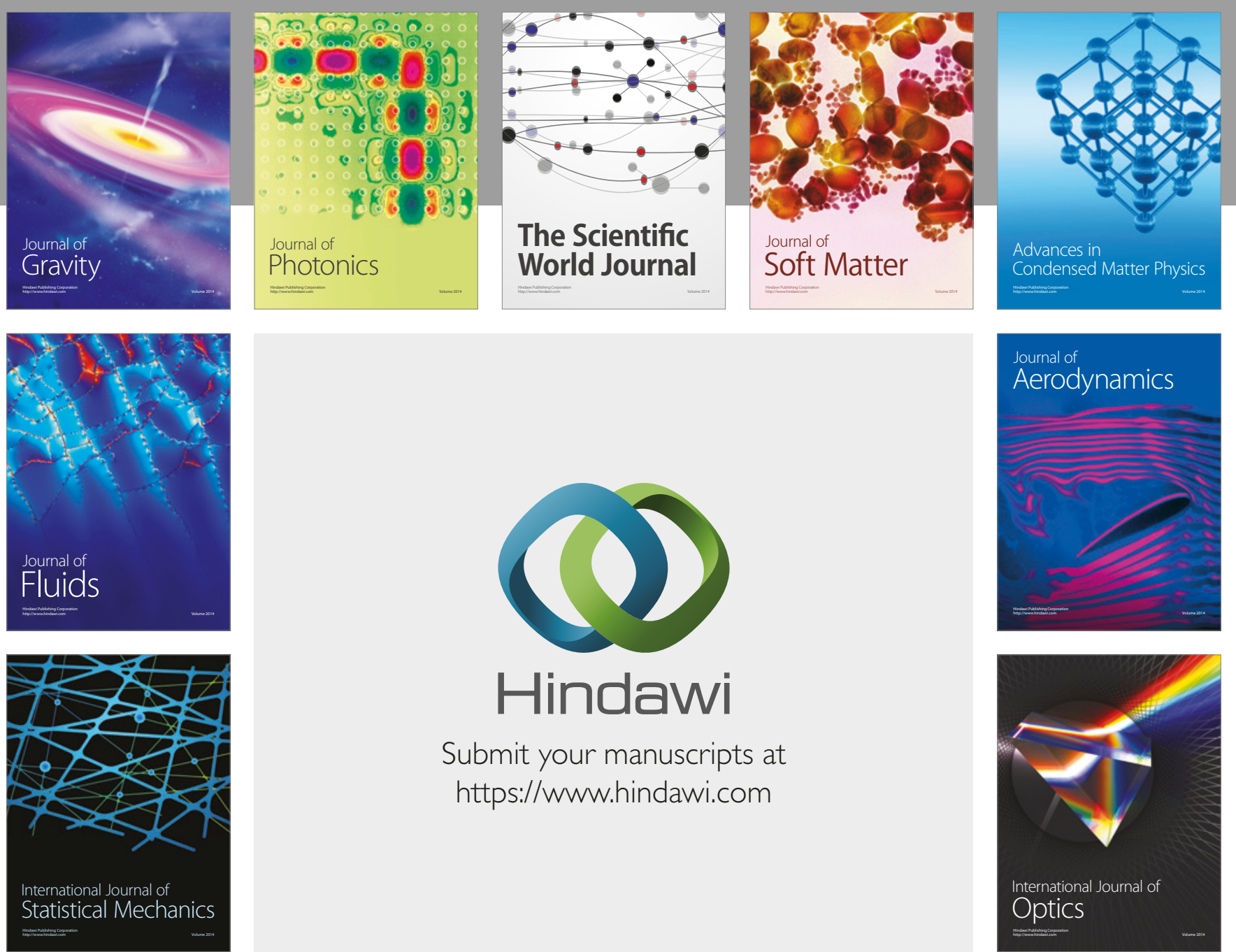

Submit your manuscripts at

https://www.hindawi.com
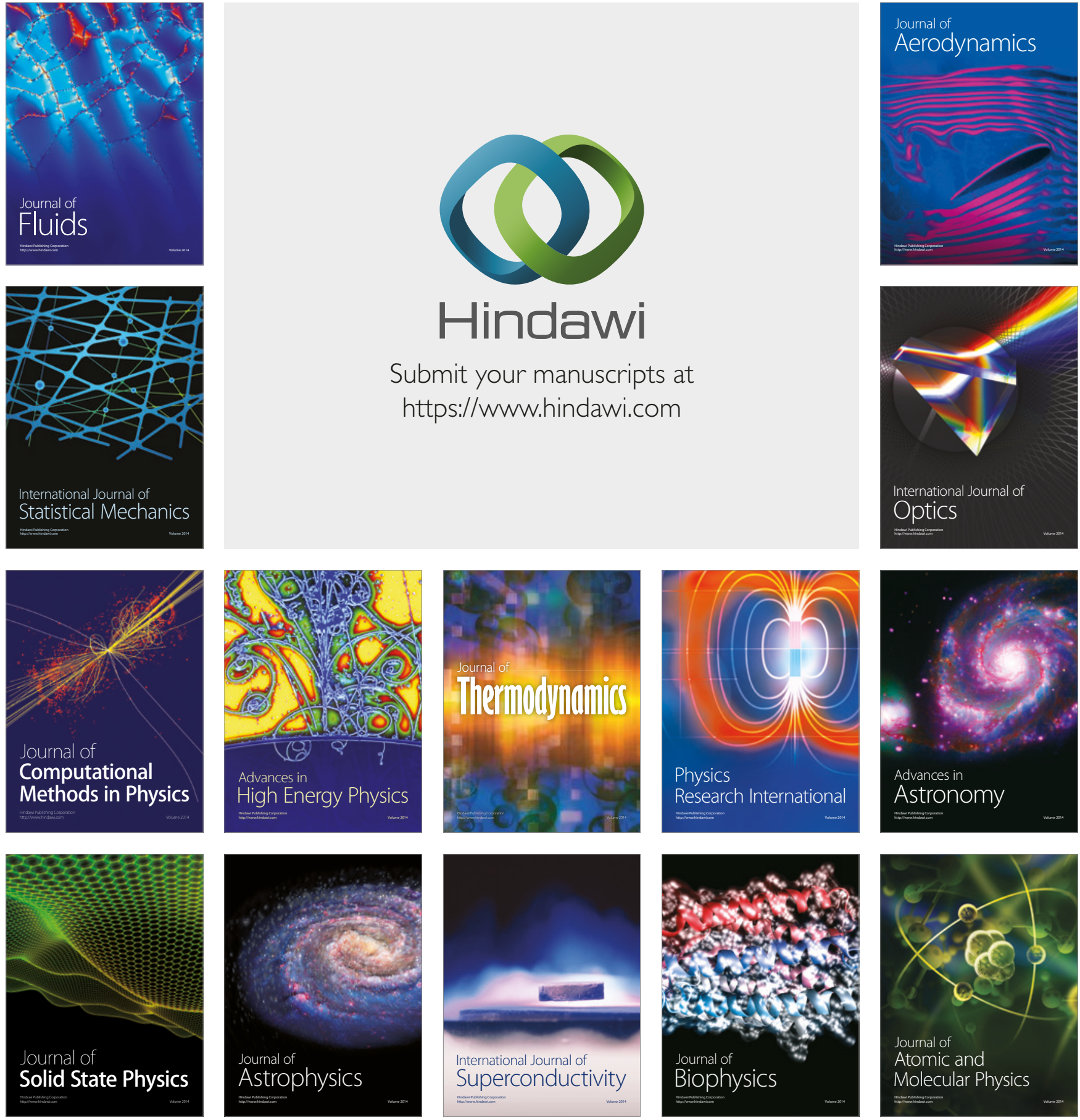